\title{
Nivel del síndrome de Burnout en docentes durante tiempos de Covid 19, región Amazonas, Perú
}

\section{Level of Burnot syndrome in teachers during times of Covid 19, Amazon region, Peru}

\author{
Marvin Guevara Vargas ${ }^{1}$, Sonia Celedonia Huyhua Gutierrez ${ }^{2}$
}

\section{RESUMEN}

El estudio tuvo como objetivo determinar el nivel de síndrome de Burnout que presentan los docentes de la Institución Educativa Emblemática "San Juan de la Libertad" del distrito de Chachapoyas, región Amazonas, Perú, 2021. Se empleo una metodología cuantitativa, de tipo básico con diseño descriptivo, con una muestra constituida por 60 docentes, el muestreo fue no probabilístico, se aplicó el Inventario de Burnout de Maslach la versión estandarizada al idioma castellano de Tacca y Tacca, (2019), la ejecución se realizó de manera virtual a través del formulario de Google y por llamada telefónica. Se encontraron que el $51.6 \%$ son del sexo femenino y el $48.4 \%$ son del sexo masculino, el $86.7 \%$ son casados y el $85 \%$ son docentes nombrados, asimismo, el $63.3 \%$ presenta un nivel bajo de Síndrome de Burnout, el 35\% nivel medio y el $1.7 \%$ nivel alto. Se concluye que la mayor parte de la plana docente encuestada ostenta niveles bajos de Síndrome de Burnout, no encontrando relación significativa entre las tres dimensiones que miden el Síndrome de Burnout con la edad, el sexo y la condición de trabajo.

Palabras clave: Síndrome de Burnout, agotamiento emocional

\begin{abstract}
The objective of the study was to determine the level of Burnout syndrome presented by the teachers of the Emblematic Educational Institution "San Juan de la Libertad" of the district of Chachapoyas, Amazonas region, Peru, 2021. A quantitative methodology was used, of a basic type with descriptive design, with a sample consisting of 60 teachers, the sampling was non-probabilistic, the Maslach Burnout Inventory was applied, the standardized version to the Spanish language of Tacca and Tacca, (2019), the execution was carried out virtually through the Google form and by phone call. It was found that $51.6 \%$ are female and $48.4 \%$ are male, $86.7 \%$ are married and $85 \%$ are appointed teachers, likewise, $63.3 \%$ present a low level of Burnout Syndrome, 35\% level medium and $1.7 \%$ high level; concluding that most of the surveyed teaching staff show low levels of Burnout Syndrome, not finding a significant relationship between the three dimensions that measure Burnout Syndrome with age, sex and work condition.
\end{abstract}

Keywords: Burnout Syndrome, emotional exhaustion

${ }^{1}$ Bachiller en Psicología de la Facultad Ciencias de la Salud de la Universidad Nacional Toribio Rodríguez de Mendoza de Amazonas. Correo electrónico: vinmar97.mg@gmail.com

${ }^{2}$ Maestra en Gerencia y Gestión de los Servicios en Salud, Enfermera. Docente adscrita de la Facultad de Ciencias de la Salud de la Universidad Nacional Toribio Rodríguez de Mendoza de Amazonas.Correo electrónico: sonia.huyhua@untrm.edu.pe 


\section{INTRODUCCIÓN}

Desde fines del año 2019 se está atravesando una dificultad sanitaria producida por la aparición de la COVID 19, en la cual la población mundial no se encontraba preparada para sobrellevar de una manera adecuada está nueva etapa, afectando en todos los ámbitos (social, económico, familiar, educación, etc.), sin embargo, si enfocamos el ámbito de la educación, donde el $100 \%$ de las instituciones educativas de los países afectados a nivel mundial tuvieron que cerrar las aulas (la presencialidad) y empezar una nueva metodología de enseñanza virtual, (Caballero, 2020) este cambio trae una adecuación inmediata y sostenible a una "nueva normalidad". La definición más aceptada hasta la actualidad del Síndrome de Burnout es de Maslach, que menciona que es: "una condición impropia de enfrentar el estrés crónico", cuyas peculiaridades trascendentales son el agotamiento emocional, la despersonalización y la disminución del desempeño personal (Saborío y Hidalgo, 2015).

Los docentes afrontan muchas complicaciones ya sean económicas o sociales, dentro de los cuales se tiene la inferioridad de remuneraciones económicas en comparación a otras profesiones, alta demanda de carga laboral, condiciones laborales inadecuadas, conflictos internos y en constate cambio en el diseño curricular de acuerdo con las nuevas exigencias que surgen cada año (Saloviita y Pakarinen, 2020).

La Organización Mundial de la Salud (OMS) en el año 2019 reconoció al Síndrome de Burnout, Síndrome de estar quemado o también conocido como desgaste ocupacional como un padecimiento crónico en la revisión número 11 del (CIE - 11), que significa clasificación estadística internacional de enfermedades y problemas de la salud conexos, la cual entraría en vigencia el 01 de enero del 2022, lo que indica la dificultad de este trastorno, enfermedad que afecta con el desempeño óptimo de los trabajadores a nivel mundial, Rodríguez et al., (2017).

\section{MATERIAL Y MÉTODO}

La investigación fue de enfoque cuantitativo de tipo básico con un diseño descriptivo. Se trabajó con la población muestral de 60 docentes. El muestreo fue no probabilístico por conveniencia Hernández et al., (2014).

Se utilizó las pruebas estandarizadas e inventarios. Asimismo, se empleó una ficha sociodemográfica y la escala de medición de Burnout de Maslach, la versión estandarizada al idioma castellano por Tacca y Tacca, (2019), en donde al realizar un análisis de confiabilidad interna obtuvieron los valores de 0.77 para agotamiento emocional, 0.66 para la dimensión de despersonalización y 0.72 para la dimensión de realización personal. El instrumento utilizado tenía tres dimensiones:

$\checkmark$ Dimensión de agotamiento emocional: Se valoro las experiencias que presenta el individuo de presentarse cansado emocionalmente ante las demandas del trabajo.

$\checkmark$ Dimensión de despersonalización: Se evaluó los índices de los individuos respecto a algunas actitudes como la frialdad y distanciamiento.

$\checkmark$ Dimensión de realización personal: Se midió la eficacia del individuo al momento de realizar sus actividades laborales su centro de trabajo.

\section{RESULTADOS}

\section{Figura 1}

Nivel de Síndrome de Burnout en los docentes de la Institución Educativa Emblemática "San Juan de la Libertad”, Distrito Chachapoyas, 2021.

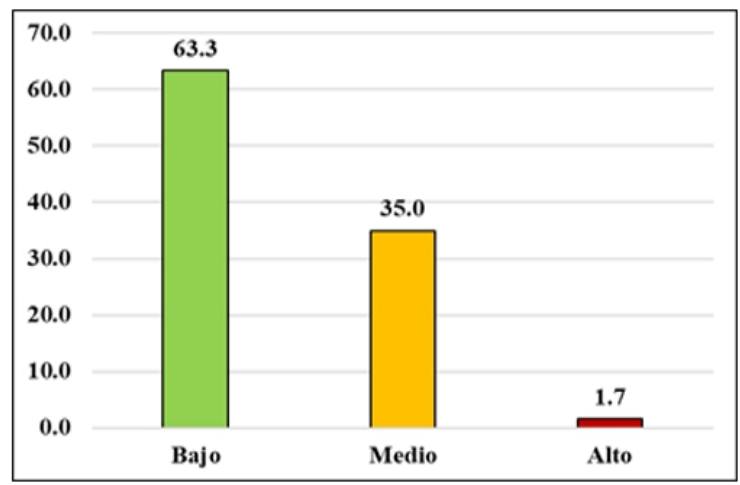

En la figura 1, se observa que el 63.3\% (38) docentes de la Institución Educativa Emblemática San Juan de la Libertad presentan un nivel bajo de Síndrome de Burnout; el 35\% (21) ostentan un nivel medio y finalmente el 1.7\%(01) ostentan un nivel alto.

\section{Tabla 1}

Nivel de agotamiento emocional según su condición laboral de los docentes, Institución Educativa Emblemática "San Juan de la Libertad", distrito Chachapoyas, 2021.

\begin{tabular}{ccccc}
\hline \multirow{2}{*}{ Nivel } & \multicolumn{4}{c}{ Condición laboral } \\
\cline { 2 - 5 } & Nombrado & $\%$ & Contratado & \% \\
\hline Bajo & 33 & 55.0 & 8 & 13.3 \\
Medio & 13 & 21.7 & 0 & 0 \\
Alto & 5 & 8.3 & 1 & 1.7 \\
\hline Total & $\mathbf{5 1}$ & $\mathbf{8 5 . 0}$ & $\mathbf{9}$ & $\mathbf{1 5 . 0}$ \\
\hline
\end{tabular}

$\mathrm{X}^{2}=2.962 ; \mathrm{GL}=2 ; \mathrm{p}=0.227$ 
En la tabla 1, se observa que el 55\% (33) corresponden a docentes nombrados que adquirieron un nivel bajo de agotamiento emocional; el 21.7\% (13) de docentes nombrados un nivel medio; el $13.3 \%$ (08) de los docentes contratados obtuvo un nivel bajo; el 8.3\% (05) de los docentes nombrados obtuvo un nivel alto y el 1.7\% (01) de los docentes contratados obtuve un nivel alto.

\section{Tabla 2}

Nivel de despersonalización según sexo, docentes de la Institución Educativa Emblemática "San Juan de la Libertad” Distrito Chachapoyas, 2021.

\begin{tabular}{lcccccc}
\hline & \multicolumn{3}{c}{ Sexo } & \multicolumn{3}{c}{ Total } \\
Nivel & F & $\%$ & $\mathrm{M}$ & $\%$ & $\mathrm{n}^{\circ}$ & $\%$ \\
\hline Bajo & 23 & 38.3 & 15 & 25.0 & 38 & $\mathbf{6 3 . 3}$ \\
Medio & 5 & 8.3 & 7 & 11.7 & 12 & $\mathbf{2 0 . 0}$ \\
Alto & 3 & 5.0 & 7 & 11.7 & 10 & $\mathbf{1 6 . 7}$ \\
\hline Total & $\mathbf{3 1}$ & $\mathbf{5 1 . 6}$ & $\mathbf{2 9}$ & $\mathbf{4 8 . 4}$ & $\mathbf{6 0}$ & $\mathbf{1 0 0 . 0}$ \\
\hline
\end{tabular}

$\mathrm{X}^{2}=3.555 ; \mathrm{GL}=2 ; \mathrm{p}=0.169$

En la tabla 2, el 38.3\% (23) de la población docente femenina se encuentran en un nivel bajo de despersonalización, el 25\% (15) docentes del sexo masculino se encuentran en un nivel bajo; el 11.7\% (7) de los docentes del sexo masculino presentan un nivel medio, al igual que en el nivel alto, el 8.3\% (5) de docentes del sexo femenino obtuvieron un nivel medio y el 5\% (3) de docentes de sexo femenino obtuvieron un nivel alto.

\section{Tabla 3}

Nivel de realización personal, según edad de los docentes, Institución Educativa Emblemática "San Juan de la Libertad" Distrito Chachapoyas, 2021.

\begin{tabular}{ccccccccc}
\hline Edad & \multicolumn{10}{c}{ Nivel derealizaciónpersonal } \\
(años) & Bajo & \% & Medio & $\%$ & Alto & $\%$ & Total & $\%$ \\
\hline $25-29$ & 0 & 0 & 0 & 0 & 2 & 3.3 & 2 & 3.4 \\
$30-39$ & 2 & 3.3 & 1 & 1.7 & 1 & 1.7 & 4 & 6.7 \\
$40-44$ & 3 & 5 & 1 & 1.7 & 2 & 3.3 & 6 & 10 \\
$45-49$ & 1 & 1.7 & 3 & 5 & 6 & 10 & 10 & 16.7 \\
$50-54$ & 1 & 1.7 & 5 & 8.3 & 5 & 8.3 & 11 & 18.3 \\
$55-59$ & 1 & 1.7 & 9 & 15 & 10 & 16.7 & 20 & 33.4 \\
$60 \mathrm{a}+$ & 0 & 0 & 1 & 1.7 & 6 & 10 & 7 & 11.7 \\
\hline Total & 8 & 13.4 & 20 & 33.3 & 32 & 53.4 & $\mathbf{6 0}$ & $\mathbf{1 0 0}$ \\
\hline
\end{tabular}

$\mathrm{X}^{2}=19.402 ; \mathrm{GL}=12 ; \mathrm{p}=0.79$

En la tabla 3, se observa que el 16.7\% (10) del grupo etario de entre 55 a 59 años, $10 \%$ (6) de 60 años, $10 \%$ (06) de 45 a 49 años, $8.3 \%$ (05) de 50 a 54 años, 3.3\% (02) de 25 a 29 años, 3.3\% (02) de 40 a 44 años y el
$1.7 \%$ (01) de 35 a 39 años, se encuentran en un nivel alto de realización personal; de igual manera se evidenció que el 15\% (09) de 55 a 59 años, $8.3 \%$ (05) de 50 a 54 años, $5 \%$ (03) de 45 a 49 años , $1.7 \%$ (01) de 35 a 39 años, $1.7 \%(01)$ de 40 a 44 años, $1.7 \%(01)$ se encuentran en un nivel medio y por último el $5 \%(03)$ de 40 a 44 años, 3.3\% (02) de 35 a 39, 1.7\% (01) de 45 a 49 años, $1.7 \%$ (01) de 50 a 54 años, $1.7 \%$ (01) de 55 a 59 años se encontraron en un nivel bajo de dicha dimensión.

\section{DISCUSIÓN}

De acuerdo, a los resultados conseguidos, donde el $63.3 \%$ de los docentes ostentan un nivel bajo de Síndrome de Burnout, similares resultados obtuvieron García et al., (2020) quienes encontraron niveles bajos al Síndrome de Burnout, esto contrasta que existe una congruencia con los resultados mostrados en esta investigación. Sin embargo, Salcedo et al., (2020) obtuvo resultados distintos, donde el $82.2 \%$ de su población ostentan un nivel medio, $16.8 \%$ un nivel alto y el $0.9 \%$ nivel bajo, también la investigación realizada en la ciudad de Lima por Medina y Ruiz (2021), en la cual encontraron que el nivel de Burnout en los docentes es alto, haciendo énfasis en el sexo femenino con un $98.8 \%$ de ellas que presentaron niveles altos a comparación con los varones $97.3 \%$. Estos resultados muestran diferencias con los conseguidos en este estudio, por ende, se diría que existe mucha influencia del lugar donde se encontraron laborando los docentes.

Dorantes, (2020) encontró mayores porcentajes de predominancia en el índice medio de Síndrome de Burnout en la plana docente femenina que en relación a sus pares del sexo masculino. Esto difiere con las derivaciones obtenidas del actual estudio donde el $51.6 \%$ de la población fue del sexo femenino y el $48.4 \%$ del sexo masculino y al no encontrar una diferencia significativa con lo que se describe al porcentaje de la población según su sexo, y al procesar los resultados conseguidos en la indagación se localizó que los docentes del sexo masculino ostentan mayor índice de Síndrome de Burnout en el nivel medio.

Dentro de la dimensión de agotamiento emocional se obtuvo que el $68.3 \%$ de la población docente entre nombrados y contratados obtuvieron un nivel bajo con lo que concierne a esta dimensión. De acuerdo con Maslach y Leiter, (2016) hace referencia que la presente dimensión es el itinerario por excelsitud para la presencia de Síndrome de Burnout, caracterizado por la sobre exigencia de este careciendo de los mecanismos necesarios para afrontar dicha situación. De igual forma, el 21.7\% consiguió un nivel medio y por último el $10 \%$ se localiza en un nivel alto. 
Con lo que concierne a la dimensión de despersonalización donde el $63.3 \%$ tiene un nivel bajo, $20 \%$ nivel medio y $16.7 \%$ un nivel alto; en la cual se debe entender que esta dimensión brota ante la carencia de recursos psicológicos y emocionales del individuo para enfrentar los desafíos que su entorno le brinda y una de sus principales manifestaciones que presenta es el cinismo y actitudes de frialdad Marsollier, (2013). Esto significa que los docentes del presente estudio no presentan en su mayoría estas actitudes negativas contra sus estudiantes, lo que facilita el buen desarrollo y manejo de sus clases virtuales. De igual manera tiene concordancia con Celio, (2021) donde el $78.3 \%$ tiene un nivel bajo; de igual manera Grijalba y Quiñones, (2021), obtuvieron que el $78.3 \%$ tiene un nivel bajo y el $65.6 \%$ tiene nivel muy leve, respectivamente, en esta dimensión lo cual podría deberse a que los docentes cuentan con un manejo adecuado de las plataformas virtuales.

En la dimensión de realización personal se obtuvo que el $53.3 \%$ de los docentes tienen nivel alto, similares resultados obtuvo Manzano, (2020) donde el $85 \%$ tiene un nivel alto, esto reflejaría que los docentes del presente estudio se encuentran motivados al momento de realizar sus clases virtuales. Gagné et al. (2010) indica que una persona que se encuentra laborando y no desarrolla sus potencialidades, no conseguirá comprometerse, ni optimizar su producción, esto contemplaría que más de la mitad de la población encuestada se encuentra motivada y en condiciones adecuadas de realizar sus clases virtuales con sus alumnos. Por otro lado, estos resultados difieren con los de Mora, (2021), que reveló que el $12.9 \%$ presentan nivel alto, lo que indicaría que estos docentes no se encontrarían motivados al máximo, esto refleja una diferencia significativa con el presente estudio.

\section{CONCLUSIONES}

La gran mayoría de la población docente encuestada exhibe niveles bajos de Síndrome de Burnout, con una representación del $63.3 \%$.

La dimensión agotamiento emocional no se encuentra relacionada con la condición laboral $(\mathrm{p}>$ $0.05)$.

La dimensión de despersonalización no se encuentra relacionada con el sexo $(\mathrm{p}>0.05)$.

La dimensión realización personal no se encuentra relacionada con la variable sociodemográfica edad ( $\mathrm{p}$ $>0.05)$.

\section{REFERENCIAS BIBLIOGRÁFICAS}

Caballero, E. (21 de abril de 2020). Manejo del estrés durante la cuarentena. Conexionesan. https://www.esan.edu.pe/apuntes empresaria les/2020/04/manejo-del-estrés laboraldurante-la-cuarentena/

Celio, J. (2021). Burnout y Satisfacción con la vida en docentes que realizan clases virtuales en un contexto de pandemia por covid-19. Puriq, 3(1),185-2012. https://orcid.org/0000 0002-8622-9919

Dorantes, J.A. (2020). El Síndrome de Burnout y su Prevalencia en las Mujeres Docentes. Praxis Investigativa Redie, 12(23), 15-31. https://dialnet.unirioja.es/servlet/articulo?co digo $=7595380$

Gagné et, al. (2010). The Motivation at Work Scale: Validation Evidence in Two Languages. Educational and Psychological Measurement,70(4), 628-646. http://sdtheory.s3.amazonaws.com/SDT/doc uments/2010_GagneForestetal_EPM.pdf

García et, al. (2020). Síndrome de Burnout y Factores Relacionados en Docentes Universitarios. Nure Investigación, 18(110), 1-12. https://www.nureinvestigacion.es/OJS/inde X.php/nure/article/view/2037/950

Grijalba, B.E. y Quiñonez, G.P. (2021). Nivel de Burnout en el trabajo remoto de los docentes en una Institución Educativa Piloto de Huancayo 2020. [Tesis de Licenciatura, Universidad César Vallejo]. https:// repositorio.ucv.edu.pe/bitstream/handle/20. 500.12692/62475/Grijalba RBE-Qui\% C3\%B1onez_EGP-SD.pdf? sequence $=1 \&$ is Allowed $=y$

Hernández et al., (2014). Metodología de la Investigación. Mcgraw-H I L L / Interamericana Editores, S.A. De C.V.

Manzano, A. (2020). Síndrome de Burnout en Docentes de una Unidad Educativa, Ecuador. Horizontes, 4(12), 499-511. Https://doi.org/ 10.33996/revistahorizontes.v4i16.132

Marsollier, R.G. (2013). La despersonalización y su incidencia en los procesos de desgaste laboral. Psicologia.com, 17(7), 1-10. http://hdl.handle.net/10401/6175

Maslach, C. y Leiter, M. (2016). Chapter 43 Burnout. En Stress: Concepts, Cognition, Emotion, and Behavior. Academic Press, 1(1), 351-357. https://doi.org/10.1002/ wps.20311

Medina, D.A. y Ruiz, B.V. (2021). Burnout y Motivación Laboral en Tiempos de 
Pandemia Covid-19 en Docentes de Instituciones Educativas de Lima Metropolitana, 2021. [Tesis de Licenciatura, Universidad César Vallejo]. https:// repositorio.ucv.edu.pe/bitstream/handle/20. $500.12692 / 62282 / \mathrm{Medina}$ RDA Ruiz_MBV-SD.pdf? sequence $=$ $1 \&$ isAllowed $=\mathrm{y}$

Mora, J.A. (2021). Sindrome de Burnout y desempeño docente en instituciones educativas del nivel secundario de los centros poblados de Chancay, 2020. [Tesis de Maestría, Universidad César Vallejo]. https://repositorio.ucv.edu.pe/bitstream/han dle/20.500.12692/55799/Mora DJASD.pdf? sequence $=1 \&$ isAllowed $=\mathrm{y}$

Organización Médica Colegial de España. (28 de abril de 2019). La OMS reconoce como enfermedad el burnout o "síndrome de estar quemado". http://www.medicosypacientes. com/articulo/la-oms-reconoce-comoenfermedad-el-burnout-o-sindrome-deestar-quemado

Rodríguez et al., (2017). Síndrome de Burnout en Docentes. IE Revista de Investigación Educativa de la REDIECH, 8(12), 45-67. https://www.redalyc.org/jatsRepo/5216/521 653267015/html/index.htm

Saborío, L. y Hidalgo, L.F. (2015). Revisión Bibliográfica: Síndrome de Burnout. Medicina Legal de Costa Rica - Edición Virtual, 32(1), S/N. https://www.scielo .sa.cr/pdf/mlcr/v32n1/art14v32n1.pdf

Salcedo et, al. (2020). Síndrome de Burnout en docentes en un contexto de emergencia sanitaria, Lima. Alpha Centuri, 1(3), 43-56. https://journalalphacentauri.com/index.php/ revista/article/view/18/21

Saloviita, T. y Pakarinem, E. (2020). Teacher burnout explained: Teacher-, student-, and organisation-levelvariables. Elsevier, 97, 114. https://www. sciencedirect. com/science/article/abs/pii/S0742051X203 14177

Tacca, D.R. y Tacca, N.L. (2019). Sindrome de Burnout y resiliencia en profesores peruanos. RIP, 22, 11-30. http://www. scielo.org.bo/pdf/rip/n22/n22_a03.pdf 